\title{
The CPW-TEC project: Planetary waves in the middle atmosphere and ionosphere
}

\author{
C. Jacobi ${ }^{1}$, N. Jakowski ${ }^{2}$, A. Pogoreltsev ${ }^{3}$, K. Fröhlich ${ }^{1}$, P. Hoffmann ${ }^{1}$, and C. Borries ${ }^{2}$ \\ ${ }^{1}$ Institute for Meteorology, University of Leipzig, Stephanstr. 3, 04103 Leipzig, Germany \\ ${ }^{2}$ DLR, Institute of Communications and Navigation, Kalkhorstweg 53, 17235 Neustrelitz, Germany \\ ${ }^{3}$ Russian State Hydrometeorological University, 98 Maloohtinsky St., Petersburg 195196, Russia
}

\begin{abstract}
Maps of vertically integrated electron density over the higher middle and polar latitudes that are regularly produced by DLR Neustrelitz are investigated with respect to planetary waves (PW) in the period range of several days. The results are compared with planetary wave analyses using stratospheric reanalyses. Case studies show that PW signatures in the ionosphere and neutral middle atmosphere waves have similar seasonal variations, indicating a possible coupling between these layers. Numerical modelling of the middle and upper atmosphere is performed to analyse the possible penetration of PW effects into the thermosphere. Numerical results show that direct propagation of PW to the thermosphere is not possible, and indirect ionospheric effects must be responsible for the wave coupling of the atmospheric layers. In the paper an overview of the data used and the methods applied in this project is given.
\end{abstract}

\section{Introduction}

Atmospheric planetary waves (PW) in the middle atmosphere are characterized by periods of 2 to 30 days (frequently called the "long-period range"). Usually, PW are not able to penetrate to altitudes above the lowermost thermosphere (Lastovicka, 2006). Nevertheless, oscillations with $\mathrm{PW}$ periods have been observed in the ionosphere, for example in ionospheric F-region maximum heights (Pancheva et al., 2002) or electron densities (Altadill et al., 2001, 2003). The simultaneous occurrence of PW in the ionosphere and stratosphere might be an indicator for vertical coupling between the middle atmosphere and the thermosphere/ionosphere system. However, the mechanisms that lead to such kind of coupling necessarily must be indirect.

Correspondence to: C. Jacobi

(jacobi@uni-leipzig.de)
There already exist some ideas about how PW may penetrate into the ionosphere. Pancheva et al. (2002) suggested a modulation of upward propagating tides through PW, or PW associated neutral wind variations inducing electric fields, which modulate the height of the maximum electron density hmF2 or the plasma density of the ionospheric F-region. Other possible mechanisms (see e.g. Altadill et al., 2001) that may lead to PW signatures in the ionosphere are the modulation of gravity waves $(\mathrm{GW})$ by $\mathrm{PW}$ in the middle atmosphere. These GW may propagate to the ionosphere, and break there, thus transporting the PW signature to the upper atmosphere. Also possible is that winds in the mesopause region may cause a redistribution of major atmospheric components in the lower thermosphere and changes the mean velocity of mass transport. This affects the vertical transport of minor constituents and provides phase changes of the electron density in the E and F region (Mikhailov, 1983). Pancheva and Lysenko (1988) proposed that PW mesopause region winds may drive the atmospheric dynamo. Altadill et al. (2001) concluded from the result of case studies that the mechanisms which lead to PW like oscillations in the ionosphere are different from case to case.

To comprehensively investigate the possible coupling between the neutral middle atmosphere and the ionosphere at PW time scales, within the CPW-TEC (Climatology of Planetary Waves seen in TEC) project we make use of maps of Total Electron Content (TEC) in a vertical air column of the ionosphere. The analysis of these maps suggests signatures of PW in the ionosphere, so that the results obtained are compared with stratospheric wave analyses accompanied by numerical model experiments on PW propagation. The project is run in collaboration between DLR-IKN Neustrelitz, Leipzig University, and the Russian State Hydrometeorological University, St. Petersburg. In the following sections an overview of the data used and the methods applied in this project is given. 


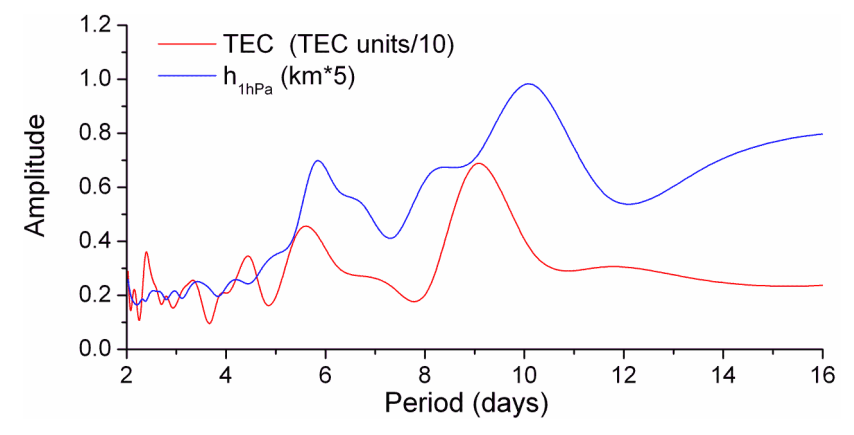

Fig. 1. Examples of amplitude spectra of GPS TEC and Met Office $1 \mathrm{hPa}$ geopotential heights over Europe, 1 September - 20 November 2004.

\section{Data base and analysis}

TEC maps are regularly produced at DLR Neustrelitz using ground based GPS measurements available from the International GPS Service (e.g. Jakowski, 1996; Jakowski et al., 2002; w3swaci.dlr.de/index.htm). Maps covering the European sector are available since 1995, while similar maps from $50^{\circ} \mathrm{N}$ to the pole are produced since 2002 . The TEC values are mapped and blended into a TEC model, which was established by the DLR (Jakowski et al., 1996) especially for TEC map construction. Here we use the results of this data assimilation from the pole to $50^{\circ} \mathrm{N}$. These TEC maps are processed by DLR Neustrelitz with a time resolution of ten minutes and an accuracy of 1-2 TEC units (1 TEC unit = $10^{16}$ electrons $/ \mathrm{m}^{2}$ ).

For the analysis of stratospheric PW we use UK Met Office assimilated data (Swinbank and O'Neill, 1994) at $1 \mathrm{hPa}$. To avoid disturbances through auroral processes in this analysis we restrict ourselves to the analysis of data at $52.5^{\circ} \mathrm{N}$. An example of amplitude spectra of GPS TEC and stratospheric $1 \mathrm{hPa}$ geopotential heights is shown in Fig. 1. The time window analysed here (1 September - 20 November 2004) has been chosen because a quasi 6-day oscillation has been detected in mesopause region radar winds (not shown here), so that autumn 2004 was chosen as a sort of first test whether long-period oscillations may be visible in both middle atmosphere and ionosphere. Oscillations at periods of 5-8 days are frequently found in the middle atmosphere under equinox conditions (e.g. Jacobi et al., 1998), so from this point of view the selected time interval can be considered as typical. Similarly, in Fig. 1 there are peaks at a period close to 6 days in both the stratosphere and ionosphere spectra. A TEC oscillation with a 9-day period is accompanied by a broad spectral maximum in the stratospheric geopotential height amplitudes, but it remains questionable whether this can be considered as the signature of middle atmosphereionosphere coupling. Periods of 6 and 9 days may be owing to the Rossby quasi 5 and 10-day waves. However, from simple spectra alone the global structure of the underlying waves cannot be revealed.

To investigate and interpret the planetary scale disturbances, we have to separate them into the travelling and standing PW components (Hayashi, 1971; Schäfer, 1979). At first, the amplitudes and phases for the zonal harmonics $m=1,2,3$ at $52.5^{\circ} \mathrm{N}$ are estimated by using the singular value decomposition (SVD) algorithm. The hourly results for TEC are daily averaged. A pair of time series is constructed representing two eastward and westward moving waves, those signals are registered at two zonal grid points with a distance equal to the phase shift between the sine and cosine functions divided by the zonal wavenumber. The coefficients for the eastward and westward travelling waves are calculated by using the phase-difference method (Pogoreltsev et al., 2002) for both waves. The Fourier amplitudes and phases of the two components are calculated for a time segment of 48days. After this, the procedure is repeated while shifting the window by one day to study the time dependency of wave activities during 2004. To filter waves the spectral information at specific period bands are combined and transformed back into the time domain. For this resulting time series a wavelet amplitude spectrum is calculated.

When analysing wavelike structures in the ionosphere by TEC or electron density data one has to keep in mind that the thermosphere is only ionized to a minor degree even at F2 layer heights. Thus, wavelike variations in TEC or electron density usually trace processes propagating in the thermosphere via interaction of neutral gas and charged particles. Consequently, PW like periods should be related either to intrinsic PW type waves in the thermosphere or corresponding modulations of other wave types such as atmospheric GW. A direct modulation of the plasma density could be induced by upward propagating electric field variations excited/modulated by planetary waves which still exist in lower thermospheric heights. Therefore, the term "ionospheric PW" is, strictly speaking, not correct. Nevertheless, for convenience we shall subsequently use this term, but have to keep in mind that it does not describe an intrinsic PW in the ionospheric plasma.

\section{Some results}

Figure 1 indicates that long-period oscillations at times may be visible in both the middle atmosphere and the ionosphere, although corresponding peaks in the spectra cannot be considered as a proof of coupling processes and may be accidental. To analyse possible corresponding wave activity in the quasi 5- and 10-day range in more detail, in Fig. 2 time series of the mean amplitudes of waves with wavenumber 1-3 (eastward and westward) in the period range 3-7 days are shown, as a representation of the quasi-5-day oscillation from Fig. 1. The stratospheric waves show a clear prevalence of westward travelling waves with wavenumber $m=1$ and, at times, $m=2$. This is also visible for the TEC data, however, 

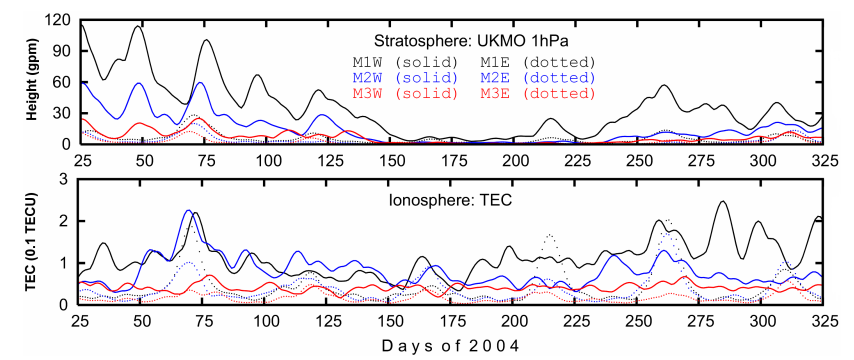

Fig. 2. Time series of wavenumber 1-3 westward (M1W, M2W, M3W) and eastward (M1E, M2E, M3E) travelling wave amplitudes for $1 \mathrm{hPa}$ geopotential height (in gpm) and TEC (in 0.1 TEC units) in the period window 3-7 days.

correlation between the stratospheric and ionospheric westward waves is weak in most part of the year, except few time intervals, e.g. around day \#75, and in autumn. Occasionally a 5-day eastward wave is visible in TEC, which in some cases is connected with a weak eastward wave in the stratosphere also.

The corresponding signature of a 10-day wave is shown in Fig. 3. As is the case with the 3-7 day oscillations in Fig. 2, the well-known seasonal cycle of stratospheric PW with maximum activity in winter and weak activity in summer is, to a certain degree, also visible in TEC. The correspondence between stratospheric and ionospheric wave activity is not strong, but still some corresponding peaks of activity can be seen, e.g. in spring for westward travelling $m=1$ waves.

Note that the large summer values of TEC amplitudes around day 200 are accompanied by a geomagnetic storm which may disturb PW signatures in TEC. Here we did not analyse a possible influence of geomagnetic variations on the ionosphere which may give rise to long-period ionospheric variations also at time scales of PW. On the other hand, PW induced oscillations of winds in the dynamo region may lead to geomagnetic oscillations at periods of PW (e.g. Kohsiek et al., 1995; Jarvis, 2006), so that in turn the external forcing of the geomagnetic field carefully has to be distinguished from PW forcing. Detailed analyses of external influence is a topic for further research.

Apart from the seasonal cycle at times there is a correspondence between the variation of wave activity both in the stratosphere and ionosphere, so, for example, in the westward propagating wave 1 around days 100 and 125 in Fig. 3 and after day 250 in Fig. 2. This correspondence leads to the conclusion that there is a possible coupling between the middle atmosphere and ionosphere. However, this correspondence is not continuously apparent during 2004, so that coupling processes between the atmospheric layers, if they exist, are variable, and intermittent.

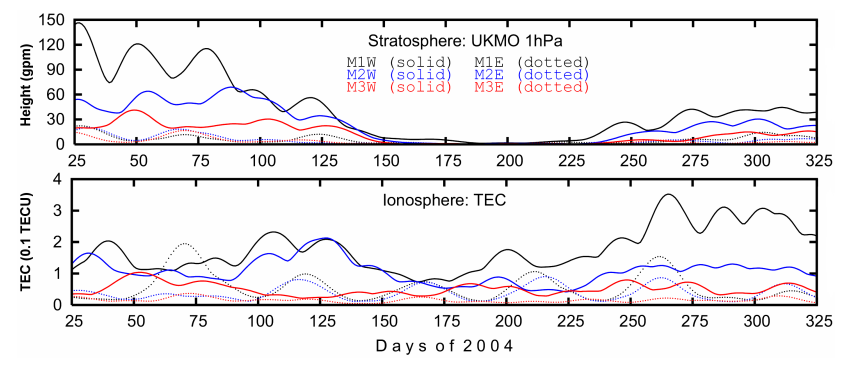

Fig. 3. As in Fig. 2, but for the 7-12 day period range.

\section{Numerical modelling}

The Middle and Upper Atmosphere Model (MUAM) is a 3D mechanistic model of the atmospheric circulation extended from the $1000 \mathrm{hPa}$ surface up to the heights of the ionospheric F2-layer. It is based on the Cologne Model of the Middle Atmosphere-Leipzig Institute for Meteorology (COMMA-LIM, Fröhlich et al., 2003). The MUAM is a gridpoint model with horizontal (latitude/longitude) resolution of $5 \times 5.625^{\circ}$, and with up to 60 levels spaced evenly in the nondimensional log-pressure height (scale height $x=-\ln \left(p / p_{0}\right)$, $p_{0}=1000 \mathrm{hPa}$ ] with a constant step size of about 0.4 . The upper boundary is placed at $x=24$ which corresponds to the geopotential height of $300-400 \mathrm{~km}$ depending on thermospheric temperature. The PW to be analysed can be explicitly forced in the model.

Additionally to the standard radiative scheme used in the COMMA-LIM version (Fröhlich et al., 2003) the EUV heating in the thermosphere has been included. Solar fluxes and absorption coefficients for each EUV spectral interval and each constituent were calculated using the model proposed by Richards et al. (1994). The constant value of 0.366 for the EUV heating efficiency has been used as recommended by Roble (1995).

To integrate the prognostic equations, the initial Cauchy problem was splitted (Marchuk, 1967; Strang, 1968) into a set of simpler problems according to the physical processes considered, which in our case are vertical diffusion of momentum and heat on one side, and all other processes on another side. Finally, we use the Matsuno (1966) timeintegration scheme with a time step of $100 \mathrm{~s}$. This was necessary because the leap-frog scheme used in COMMA-LIM becomes unstable in the upper thermosphere where viscous and thermal conduction terms are dominant.

First results of numerical modelling of planetary waves are shown in Fig. 4. We consider here the stationary wave, and the westward propagating 10-day wave as examples. Other waves will be analysed in future investigations. In Fig. 4, zonal wind amplitudes of the stationary PW and the westward propagating 10-day wave are presented. As background conditions those of January had been chosen. Note that the waves do not propagate to the thermosphere, which shows 

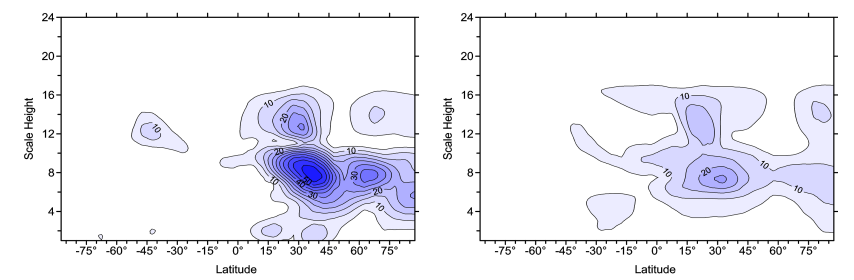

Fig. 4. Zonal wind amplitudes of the stationary PW (left panel) and the westward propagating wavenumber 1 Rossby 10-day wave (right panel), as modelled with the MUAM model for January conditions.

that a direct propagation of waves to the ionosphere is not possible as expected. Similarly, the solar tides only propagate to the lowermost thermosphere (upper panels of Fig. 5). The strong amplitudes in the thermosphere are not owing to propagating tides, but to in situ forcing of the thermosphere in the course of the day. This can be deduced from the phase behaviour of the diurnal tide, which clearly changes above a height of about 18 scale heights. For the semidiurnal tide, the change in phase behaviour is not so clearly expressed, but there is still a tendency visible that for heights above about 21 scale heights no further phase change with height occurs, and thus the semidiurnal oscillations there are due to in situ heating of the thermosphere.

\section{Conclusions}

We have shown, using Northern Hemisphere TEC data as well as stratospheric reanalyses that there probably exist coupling processes that lead to corresponding bursts of PW activity in the middle atmosphere and ionosphere, especially at periods and wavenumbers known as typical for the quasi-5 and 10-day PW. This coupling is obviously intermittent, and the underlying processes are not yet known. A numerical modelling experiment using an essentially neutral middle atmosphere model shows that both PW and tides do not propagate to the ionospheric F-region, so that direct PW propagation as well as tidal modulation through PW, according to these first results, may be ruled out as candidates for the coupling process. However, the numerical model at this stage only includes prescribed ionospheric electron densities and fixed magnetic fields, so that electrodynamic processes are not included in detail. In addition, the MUAM model still includes an improved Lindzen-type GW parameterisation (see Jacobi et al., 2006, and references therein) that is not specifically designed to describe those GW that are able to propagate to and force corresponding travelling ionospheric disturbance propagation in the thermosphere/ionosphere. Especially the question of the wavelength and frequency spectrum of those waves, and how this can be incorporated in a parameterisation, still has to be considered. Therefore, at this stage the question of coupling processes still remains open.
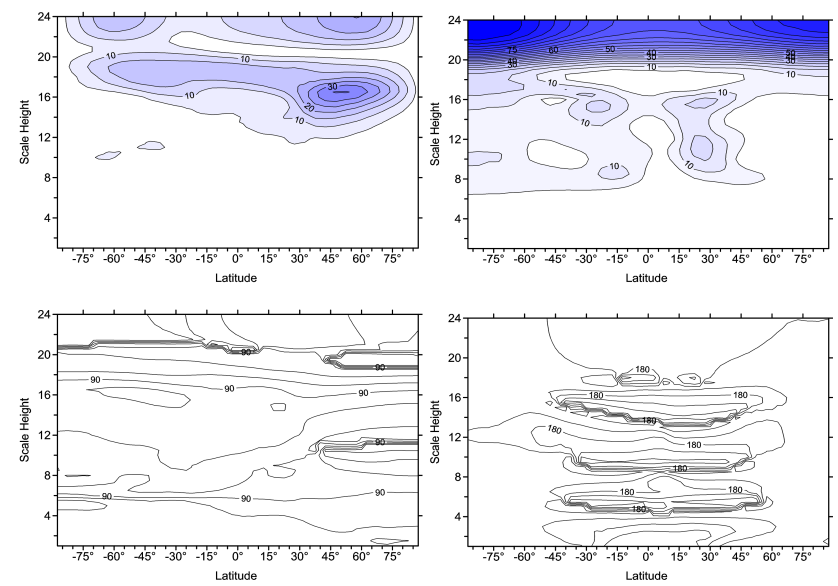

Fig. 5. Amplitudes (upper panels) and phases (lower panels, $360^{\circ}$ equal $24 \mathrm{~h}$ ) of the semidiurnal (left panels) and diurnal tide (right panels), as modelled with the MUAM model for January conditions.

Acknowledgement. This study was supported by Deutsche Forschungsgemeinschaft under JA 640/3-1 and JA 836/19-1 within the special priority program SPP 1176 "CAWSES" and by RBFR under grant RFBR-DFG 05-05-04001. Stratospheric Reanalyses have been provided by UK Met Office through BADC.

\section{References}

Altadill, D., Apostolov, E. M., Sole, J. G., and Jacobi, C.: Origin and development of vertical propagating oscillations with periods of planetary waves in the ionospheric F region, Phys. Chem. Earth (C), 26, 387-393, 2001.

Altadill, D., Apostolov, E. M., Jacobi, Ch., and Mitchell, N. J.: 6day westward propagating wave in the maximum electron density of the ionosphere, Ann. Geophysicae, 21, 1577-1588, 2003.

Fröhlich, K., Pogoreltsev, A., and Jacobi, Ch.: Numerical simulation of tides, Rossby and Kelvin waves with the COMMALIM model, Adv. Space Res., 32, 863-868, doi:10.1016/S02731177(03)00416-2, 2003.

Hayashi, Y.: A general method of resolving disturbances into progressive and retrogressive waves by space Fourier and time crossspectral analyses, J. Meteor. Soc. Jpn., 49, 125-128, 1971.

Jacobi, Ch., Schminder, R., and Kürschner, D.: Planetary wave activity obtained from long-term (2-18 days) variations of mesopause region winds over Central Europe $\left(52^{\circ} \mathrm{N}, 15^{\circ} \mathrm{E}\right), \mathrm{J}$. Atmos. Solar-Terr. Phys., 60, 81-93, 1998.

Jacobi, Ch., Fröhlich, K., and Pogoreltsev, A.: Quasi two-day-wave modulation of gravity wave flux and consequences for the planetary wave propagation in a simple circulation model, J. Atmos. Solar-Terr. Phys., 68, 283-292, 2006.

Jakowski, N.: TEC monitoring by using satellite positioning systems, in: Modern Ionospheric Science, edited by: Kohl, H., Rüster, R., and Schlegel, K., EGS, Katlenburg-Lindau, ProduServ GmbH Verlagsservice, Berlin, 371-390, 1996.

Jakowski, N., Heise, S., Wehrenpfennig, A., Schlüter, S., and Reimer, R.: GPS/GLONASS-based TEC measurements as a con- 
tributor for space weather forecast, J. Atmos. Solar-Terr. Phys., 64, 729-735, 2002.

Jarvis, M. J.: Planetary wave trends in the lower thermosphere Evidence for 22-year solar modulation of the quasi 5-day wave, J. Atmos. Solar-Terr. Phys., 68, 1902-1912, 2006.

Kohsiehk, A., Glassmeier, K., and Hirooka, T.: Periods of planetary waves in geomagnetic variations, Ann. Geophysicae, 13, 168176, 1995.

Lastovicka, J.: Forcing of the ionosphere by waves from below, J. Atmos. Solar-Terr. Phys., 68, 479-497, 2006.

Marchuk, G. I.: Numerical Methods in Weather Prediction (in Russian), Gidrometeoizdat, Leningrad, 1967 (English edn., Academic Press, New York, 1974).

Matsuno, T.: A finite difference scheme for time integrations of oscillatory equations with second order accuracy and sharp cutoff for high frequencies, J. Meteorol. Soc. Jpn., 44, 76-84, 1966.

Mikhailov, A. V.: Mechanism of in phase variations of electron concentration between E and F2 ionospheric layers (in Russian), Geomagn. Aeronomy, 23, 557-561, 1983.

Pancheva, D. and Lysenko, I.: Quasi-two-day fluctuations observed in the summer F-region electron maximum, Bulg. Geophys. J., 14, 41-51, 1988.

Pancheva, D., Mitchell, N., Clark, R. R., Drobjeva, J., and Lastovicka, J.: Variability in the maximum height of the ionospheric F2-Layer over Millstone Hill (September 1998 - March 2000); influence from below and above, Ann. Geophysicae, 20, 18071819, 2002.
Pogoreltsev, A. I., Fedulina, I. N., Mitchell, N. J., Muller, H. G., Luo, Y., Meek, C. E., and Manson, A. H.: Global free oscillations of the atmosphere and secondary planetary waves in the MLT region during August/September time conditions, J. Geophys. Res., 107, 4799, doi:10.1029/2001JD001535, 2002.

Richards, P. G., Fennelly, J. A., and Torr, D. G.: EUVAC: A solar EUV flux model for aeronomic calculations, J. Geophys. Res., 99, 8981-8992, 1994. (correction, J. Geophys. Res., 99, 13283 , 1994).

Roble, R. G.: Energetics of the mesosphere and thermosphere, in: The Upper Mesosphere and Lower Thermosphere: A Review of Experiment and Theory, Geophys. Monogr. Ser., Vol. 87, edited by: Johnson, R. M. and Killeen, T. K., 1-21, AGU, Washington, D. C., 1995.

Schäfer, J.: A space-time analysis of tropospheric planetary waves in the northern hemisphere, J. Atmos. Sci., 36, 1117-1123, 1979.

Strang, G.: On the construction and comparison of difference schemes, SIAM J. Numer. Anal., 5, 516-517, 1968.

Swinbank, R. and O'Neill, A.: A stratosphere-troposphere data assimilation system, Mon. Weather Rev., 122, 686-702, 1994. 